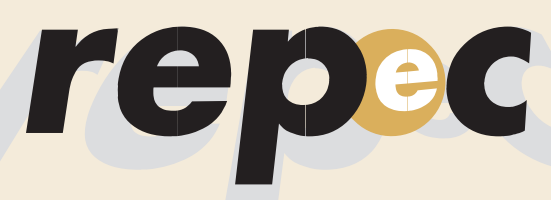

REPeC, Brasília, v.8, n.1, art. 1, p. 5-21, jan./mar. 2014 Disponivel online em www.repec.org.br

\title{
Ressonância de Artigos e Fator de Impacto de Periódicos Brasileiros de Contabilidade
}

\section{Resumo}

Este estudo buscou identificar quais características dos artigos publicados em periódicos contábeis brasileiros estão associadas à ressonância na produção científica. Tem abordagem teóricoempírica e delineamento quantitativo. A estratégia foi o levantamento e a coleta utilizou a análise do conteúdo dos resultados individuais de busca de cada artigo no Google Acadêmico. A amostra compreendeu 577 artigos publicados entre 2006 e 2011 nos 4 periódicos classificados nos estratos superiores do Qualis/CAPES 2012: Contabilidade Vista \& Revista, Revista Contabilidade \& Finanças, Revista de Contabilidade e Organizações e Revista Universo Contábil. Foram efetuados 1.655 registros categorizados em uma planilha eletrônica, sendo 1.372 citações recebidas e 283 artigos não citados. Utilizou-se o qui-quadrado e a análise de correspondência, além do cálculo do fator de impacto dos periódicos. Os achados demonstraram média de 2,38 citações por artigo e 49,05\% dos artigos não foram citados. Há associação entre periódico, ano de publicação e idioma do artigo com citações recebidas por veículo de citação, bem como entre idioma do artigo e país da citação. Os artigos da RC\&F receberam $66,33 \%$ das citações, sendo o periódico de maior peso para explicar a variabilidade dos dados. O maior fator de impacto em 2011 foi da RC\&F $(0,861)$, seguida da CVISTA $(0,667)$, RCO $(0,458)$ e UNIVERSO $(0,458)$, com média geral de 0,578 . Concluiu-se que a ressonância da produção científica analisada pode ser considerada baixa, a variabilidade dos dados possui relação com as características dos artigos/periódicos e tem diminuído a distância do fator de impacto entre os periódicos.

Palavras-Chaves: Comunicação científica, Cientometria, Fator de Impacto, Periódicos de Contabilidade

\begin{abstract}
Iracema Raimunda
Brito Neves Aragão

Doutoranda em Controladoria e

Contabilidade (USP) e professora da Universidade Estadual de Feira de Santana (UEFS). Contato: Av. Transnordestina, S/N, Campus Universitário, Novo Horizonte, Feira de Santana, BA, CEP: 44036-900. E-mail: irbn31@yahoo.com.br
\end{abstract}

José Renato Sena Oliveira Doutorando em Controladoria e Contabilidade (USP) e professor da Universidade Estadual de Feira de Santana (UEFS). Contato: Av. Transnordestina, $\mathrm{S} / \mathrm{N}$, Campus Universitário, Novo Horizonte, Feira de Santana, BA, CEP: 44036-900. E-mail: jrsenna@uefs.br

\section{Gerlando Augusto}

Sampaio Franco de Lima Livre-Docente em Ciências Contábeis (USP) e professor da Universidade de São Paulo (USP). Contato: Av. Prof. Luciano Gualberto, 908, FEA 3, Cidade Universitária, São Paulo, SP, CEP: 05508-010.

E-mail: gerlando@usp.br 


\section{Introdução}

A pesquisa é elemento determinante no processo de desenvolvimento científico e tecnológico de um país. Todavia, a disseminação e a ressonância da produção científica são aspectos que precisam ser observados a fim de que o conhecimento gerado atenda às expectativas de qualidade de vida e desenvolvimento social.

Para cientistas e filósofos, a atividade da ciência, enquanto busca de conhecimento, é em si o maior bem social e quaisquer outros benefícios sociais são apenas subprodutos dela (Nelson, 1959). Num sentido amplo, a pesquisa pode ser considerada como busca e inquirição da realidade para a elaboração de um conhecimento auxiliar à compreensão do real que norteia as ações humanas (Carmona, 2011; Czarniawska, 2011).

A comunicação científica é parte fundamental do processo de criação do conhecimento, assim, de nada valerá o esforço empreendido por meio da ação do pesquisador se os resultados da pesquisa não forem divulgados - pesquisas não divulgadas são inúteis, uma vez que não produzirão implicações e críticas, suas descobertas não serão apreciadas nem a teoria poderá ser aceita ou desprezada (Meadows, 1999; Tahai \& Rigsb, 1998).

Desde o século XVII, publicar em periódicos científicos é uma das maneiras de disseminar o conhecimento produzido (Mueller, 2000). Kuramoto (2006, p. 91) destaca que: “[...] o resultado das pesquisas científicas é divulgado à comunidade por meio de revistas. Os procedimentos para a publicação dessa informação foram estabelecidos pelo sistema de comunicação científica, o qual vem se consolidando ao longo de mais de três séculos".

Estudos anteriores, que discutem aspectos da pesquisa, têm analisado seus atributos à luz da cientometria da produção científica, como: fator de impacto (Packer, 2001; Strehl, 2005; Amin \& Mabe, 2007; Vanclay, 2012); citações (Garfield, 1972); e outros relativos a periódicos e elementos de produtividade de pesquisadores contábeis (Rosenstreich \& Wooliscroft, 2009; Lee, Yap, Lim, \& Tam, 2012). Por meio deles, busca-se conhecer características, bem como avaliar a qualidade e ressonância dessa produção.

Diante desse contexto, tem-se se a seguinte questão norteadora: quais características dos artigos publicados em periódicos contábeis brasileiros vinculados aos Programas de Pós-Graduação em Ciências Contábeis estão associadas à ressonância na produção científica?

Os objetivos deste trabalho buscam:

- Examinar a ressonância da produção analisada a partir da associação entre os periódicos e o volume de citações recebidas pelos artigos publicados nesses.

- Identificar o fator de impacto dos artigos analisados a partir das citações recebidas listadas no Google Acadêmico.

Do ponto de vista da cientometria, área de conhecimento que estuda medidas e índices de avaliação das pesquisas, o trabalho se justifica pela importância de observar se a produção científica contábil disseminada nos periódicos brasileiros da área encontra ressonância na produção científica, definida como o interesse dos pesquisadores pelas publicações revelado por meio da quantidade de citações recebidas (Meadows, 1999; Pinto, 2008).

Estudar a repercussão do que é publicado nos principais periódicos da área sobre as pesquisas realizadas pela comunidade científica é um meio de conhecer a percepção de validade externa da produção do conhecimento a partir das citações (Garfield, 1972; Flick, 2009; Pendlebury, 2009; Lee, Yap, Lim, \& Tam, 2012).

Ao trazer à tona a discussão sobre a ressonância da produção contábil brasileira, espera-se provocar reflexões no âmbito acadêmico quanto à repercussão e à qualidade do que se dissemina, bem como sobre a necessidade de buscar garantir critérios de validade ao conteúdo produzido e difundido nos principais periódicos da área. Espera-se que o conhecimento destes aspectos possa levar à mudança de práticas e trazer maior visibilidade e reconhecimento à produção científica contábil, evidenciados pelo fator de impacto.

Esta é a primeira seção do estudo, a Introdução. Na segunda, terceira e quarta seções, respectivamente, tem-se o referencial teórico - com tópicos sobre a disseminação, a ressonância e o fator de impacto da pesquisa científica; a metodologia e análise de resultados. Na quinta e última seção, apresentam-se as conclusões do estudo. 


\section{Referencial Teórico}

\subsection{A disseminação e a ressonância da pesquisa científica}

Os periódicos são canais relevantes de comunicação entre os pesquisadores e a comunidade (Campello, 2000), todavia, os resultados das pesquisas disseminados em periódicos científicos tornam-se, por vezes, superados em virtude do ritmo das mudanças científicas e tecnológicas. A tempestividade para publicação de pesquisas tornou-se necessária para que não se perca a oportunidade de contribuir para os avanços intelectual e social.

Um estudo importante acerca da disseminação da pesquisa foi realizado por Garfield (1972), que explica a criação do Science Citation Index (SCI), pelo Institute for Scientific Information (ISI), a partir da análise de aproximadamente um milhão de citações recebidas por textos publicados em 2.200 periódicos multidisciplinares mundiais em 1969. O SCI passou a fornecer métricas para a classificação dos periódicos segundo o seu impacto, propiciando uma busca crescente por publicações em periódicos indexados que possuem maior fator de impacto (FI) (Kuramoto, 2006).

O ISI passou a publicar anualmente três indicadores, por título de periódico: o índice de citação imediata (Immediacy Index), a meia-vida das citações (Cited Half-Life) e o índice bibliométrico fator de impacto (Impact Factor). São calculados com base nos dados de citações categorizados por periódicos, são publicados no Journal Citation Reports (JCR) e são utilizados como parâmetro de avaliação de pesquisadores e instituições (Strehl, 2005).

As referências citadas em um artigo científico expressam, em maior ou menor grau, o foco geográfico do trabalho. Por outro lado, a análise do impacto compreende a mensuração da disseminação de um conhecimento gerado dentro e fora das fronteiras do país de origem da pesquisa. Para Ladle, Todd \& Malhado (2012), há uma tendência de os pesquisadores citarem artigos que possuem resumos ou traduções na sua língua nativa, principalmente nas nações em desenvolvimento. As citações de estudos do próprio país podem decorrer da concentração de pesquisas voltadas ao seu desenvolvimento. Essa preferência fomenta o crescimento do impacto dos periódicos nativos de países vinculados ao JCR, por exemplo.

Christensen (2011) aponta que publicar um estudo não é suficiente, mesmo em periódicos de primeira linha; a pesquisa deve ter impacto e trazer reflexões inovadoras. Para Ohlson (2011), o sucesso de uma pesquisa acontece quando esta se torna conhecida e, geralmente, bastante citada. Meadows (1999) acredita que uma maneira de avaliar a qualidade de uma publicação em revistas científicas se firma no nível de interesse dos outros pela pesquisa e tal interesse pode ser mensurado por meio da quantidade de citações recebidas.

Para Chan \& Costa (2005), a estrutura brasileira de investigação e da capacidade de absorver a ciência é fraca e esse cenário tem conduzido a baixos níveis de produção científica. Os novos conhecimentos mundiais em termos de ciência são basicamente oriundos de países ricos, cujos gastos em pesquisa e desenvolvimento são elevados. A título de ilustração, em um estudo comparativo do desempenho da ciência, King (2004) verificou que Estados Unidos, Reino Unido, Alemanha, Japão e França foram responsáveis por quase 70\% das publicações científicas do mundo (1997-2001).

\subsection{Estudos Antecedentes}

Em pesquisa para compreender a função do periódico científico como veículo formal de comunicação do conhecimento e entre os pares da comunidade científica, Miranda \& Pereira (1996) analisaram revistas científicas impressas até o final dos anos 80. Tais autores destacam o periódico como meio primário de disseminação dos resultados de pesquisa e de contribuição para ampliar o conhecimento, apesar da ausência de retorno sobre como os resultados de seus trabalhos são distribuídos e qual seu impacto na comunidade científica. 
Pinto \& Andrade (1999) demonstraram o cálculo do fator de impacto de revistas científicas, bem como as consequências do número de citações de artigos científicos na literatura internacional, evidenciando sua importância e limitações. $\mathrm{O}$ artigo expõe que a discussão sobre a frequência com que um pesquisador é citado tomou visibilidade e polemizou-se quando o jornal Folha de S.Paulo (1995) publicou os nomes dos 170 pesquisadores em atividade no país que, entre 1981 e 1993, possuíam mais de duzentas citações na literatura internacional, de acordo com uma base de dados do ISI.

Yamamoto, Menandro, Koller, Lo Bianco, Hutz, Bueno \& Guedes (2002) realizaram investigação para apresentar uma nova etapa do processo avaliativo de periódicos científicos brasileiros da área da psicologia. Tais autores discutem sobre a responsabilidade de monitoração e controle da produção, para viabilizar a qualidade do periódico científico em função do conhecimento a ser disseminado.

O estudo realizado por Vilhena e Crestana (2002) enfatizou a crescente preocupação, por parte dos órgãos de fomento, em ranquear as revistas científicas e avaliar o número de citações das mesmas - seja na apreciação de projetos, para consentimento de bolsas de estudo ou mesmo na avaliação de cursos de pós-graduação - dado que, o periódico é um dos canais mais utilizados para a comunicação de pesquisa científica.

Strehl (2005) discutiu variáveis que apresentam maior influência sobre o FI e abordagens para medição da obsolescência da literatura. A autora conclui o estudo com reflexões a respeito do sistema de avaliação científica brasileiro e o papel do SciELO na formulação de indicadores bibliométricos.

Amara e Landry (2012) estudaram a avaliação de desempenho de pesquisadores de escolas canadenses de negócios a partir de dados extraídos da Thomson ISI Web of Science (WoS) ou do Google Scholar (GS). Concluíram que o desempenho médio dos pesquisadores, tomando por base o número de contribuições, citações e fator-h, é mais alto quando são avaliados usando o GS ao invés do WoS.

Wang (2012) realizou uma pesquisa com técnica bibliométrica e análise de rede social para examinar 34.764 referências citadas em 647 artigos a partir de duas revistas de contabilidade citadas no SSCI e SCI. Percebe-se que baixas co-citações dos autores denota que eles não foram susceptíveis a ter impacto significativo sobre o desenvolvimento do seu campo, ou suas publicações eram tão recentes que não possibilitavam ter qualquer impacto.

\subsection{Fator de impacto $(\mathrm{FI})$ : representatividade e mensuração}

O cálculo do FI considera o número de vezes que cada artigo é citado por outros e tem sido um indicador de qualidade da revista em que a pesquisa foi publicada. $\mathrm{O}$ cálculo desse indicador a partir do impacto das publicações na comunidade científica é denominado pela bibliometria e cientometria como análise de citações, ou estudo de citações (Strehl, 2005).

Pinto (2008) destaca que a cientometria possui duas aplicações principais: (1) avaliação da produção para repartição de verbas para pesquisa; (2) desenvolvimento de critérios de qualidade para direcionar os leitores na escolha da melhor evidência científica, a exemplo do fator de impacto dos periódicos publicado anualmente pelo ISI.

Ao estudar citações, Garfield (1972) verificou que a maior parte das referências cita relativamente poucas revistas e há predominância de citações oriundas de estudos publicados em alguns periódicos preferenciais por parte dos usuários da base. Para o autor, tal análise traz preocupações em relação ao aumento no número de revistas científicas e técnicas, pois ele não representa maior cobertura da literatura necessária às pesquisas.

O fator de impacto de um periódico em 2011 pelo modelo apresentado por Garfield (1972), por exemplo, é calculado pelo Total de citações recebidas em 2011 pelos artigos publicados em 2009 e 2010, dividido pelo Total de artigos publicados em 2009 e 2010.

Um dos aspectos criticados no FI é a falta de comparabilidade entre periódicos de diferentes áreas - textos de áreas mais amplas tendem a receber mais citações, o que, não necessariamente significará maior qualidade da produção em relação a áreas com menor número de pesquisadores e estudos menos abrangentes (Pinto, 2008). 
Amin \& Mabe (2007) dedicaram-se a estudar graficamente o acúmulo de citações de um periódico e ratificam que esse gráfico tende a seguir uma curva de aumento acentuado de citações em determinado período de tempo. Sintetizaram o modelo conforme Figura 1:

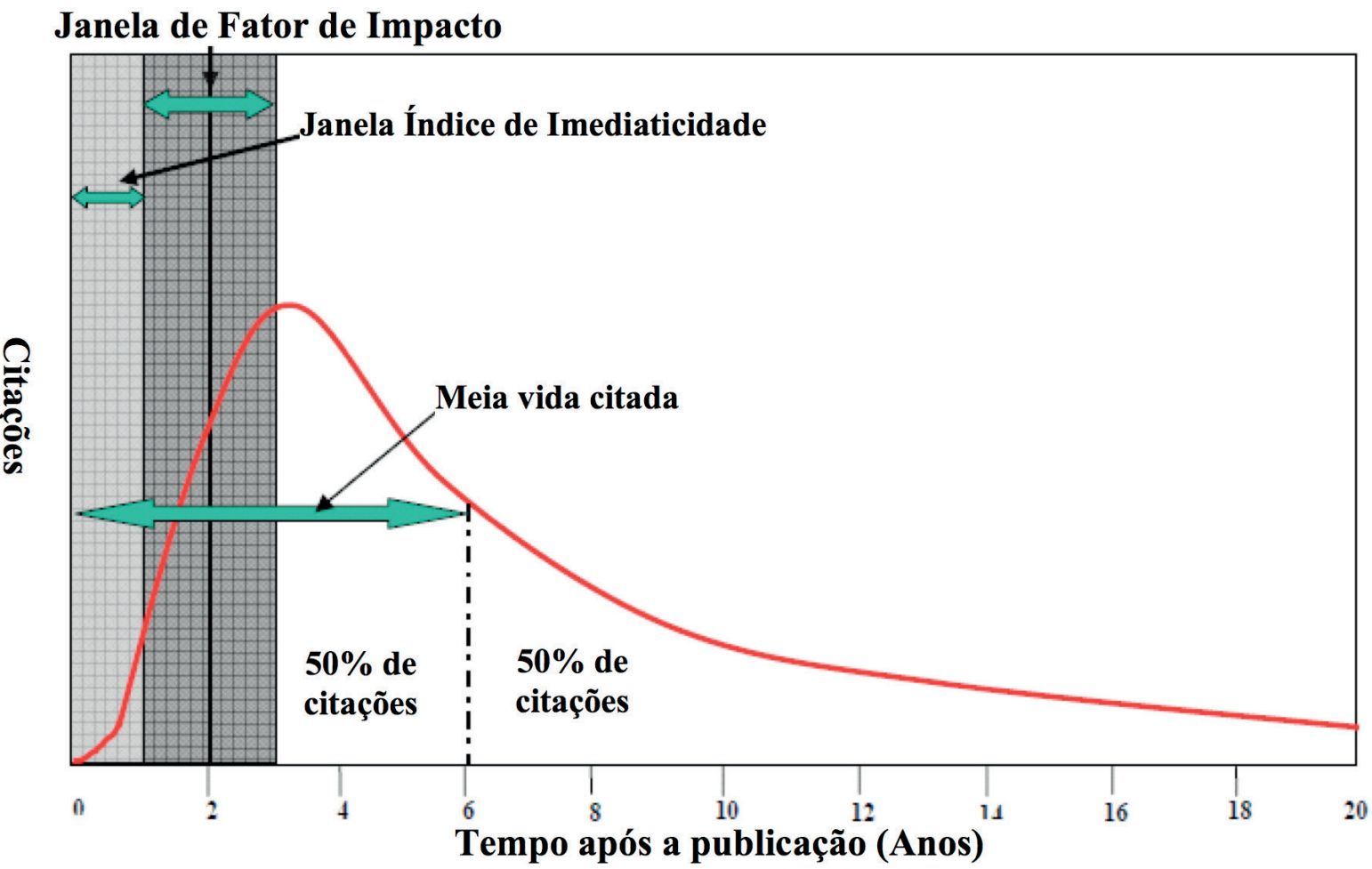

Figura 1. Curva de citação generalizada

Fonte: Amin \& Mabe (2007)

Nesse modelo os seis primeiros anos da publicação de um artigo concentram $50 \%$ das citações que esse pode receber ao longo de sua vida. O primeiro ano não é considerado no cálculo por ser um período de tempo curto para avaliação - índice imediato do acesso.

De acordo com Bianco (2004), estudos realizados pelo ISI indicam que, literalmente, o fator de impacto é o alcance do tamanho da curva de citações em dois e três anos. Geralmente, as citações de artigos publicados em um ano específico aumentam nos anos seguintes para atingir um pico entre dois e seis anos após a publicação e declinam exponencialmente a partir desse período. Ou seja, se uma publicação científica não consegue obter um grau de ressonância no prazo de dois a três anos, é improvável que isso possa acontecer num prazo superior a seis anos, visto que a partir de então essa probabilidade diminui acentuadamente.

O FI de um periódico tende a variar em função da área de conhecimento, do número de autores e do seu tamanho. Um periódico com 35 artigos por ano é considerado pequeno e é grande quando publica mais de 150 artigos anuais. Nas ciências sociais, em geral, os periódicos possuem menor fator comparados com os das ciências naturais; as comparações de FI devem ser feitas em periódicos da mesma área (Mueller, 1999; Amin \& Mabe, 2007).

De acordo com os estudos, pode-se inferir que como medida cientométrica o fator de impacto pode auxiliar a evidenciar a influência de periódicos em sua área de conhecimento, todavia, não é uma medida direta da qualidade desses. 


\section{Metodologia}

Trata-se de um estudo com abordagem teórico-empírica e delineamento quantitativo. A partir das ideias de Martins \& Theóphilo (2007), trata-se de um levantamento no que tange à estratégia de pesquisa, embora apresente também características da pesquisa documental. A técnica de coleta de dados foi a análise de conteúdo, com acesso eletrônico aos sites.

\subsection{Composição e critérios de seleção da amostra}

A amostra é composta pelos artigos publicados nos periódicos contábeis brasileiros editados por instituições que oferecem Programas de Pós-Graduação stricto sensu em Ciências Contábeis, Contabilidade e/ou Controladoria e classificados nos estratos superiores da área de Administração, Ciências Contábeis e Turismo. Tais estratos compreendem os periódicos mais relevantes de cada área definidos pelo Sistema de Avaliação de Periódicos da Coordenação de Aperfeiçoamento de Pessoal de Nível Superior Qualis/CAPES (A1, A2 e B1) na última avaliação trienal (2009-2011) (CAPES, 2012).

Foi elaborada uma lista com os 21 Programas de Pós-Graduação (PPG) stricto sensu em Ciências Contábeis, Contabilidade e/ou Controladoria reconhecidos ou recomendados no site da CAPES, tendo sido excluídas as repetições. Foram realizadas visitas aos sites das Instituições de Ensino Superior (IES) para identificação de periódico contábil vinculado ao PPG ou ao Departamento/Faculdade que oferece o Programa. Foram identificados 13 periódicos contábeis, dos quais 4 pertencem aos estratos superiores do Qualis/CAPES:

Tabela 1

Periódicos contábeis vinculados aos PPG stricto sensu em Ciências Contábeis, Contabilidade e/ou Controladoria, classificados nos estratos superiores (Qualis/CAPES 2012)

\begin{tabular}{lcclc}
\multicolumn{1}{c}{ Programa } & IES & UF & Periódico & EstratoQualis \\
\hline 1. Ciências Contábeis & FURB & SC & Revista Universo Contábil & B1 \\
\hline 2. Ciências Contábeis & UFMG & MG & Contabilidade Vista \& Revista & B1 \\
\hline 3. Controladoria e Contabilidade & USP & SP & Revista Contabilidade \& Finanças (RC\&F) & A2 \\
\hline 4. Controladoria e Contabilidade & USP/RP & SP & Revista de Contabilidade e Organizações (RCO) & B1 \\
\hline
\end{tabular}

Fonte: elaborada pelos autores a partir dos sites dos Programas e do Qualis/CAPES

Os periódicos Brazilian Business Review (BBR) e Revista Brasileira de Gestão de Negócios (RBGN), editados pela FUCAPE e UNIFECAP, pertencem aos estratos superiores A2 e B1, respectivamente. Um levantamento dos artigos publicados nesses periódicos em 2011 revelou que mais da metade pertencem a outras áreas, como Marketing e Economia. Em função dessa característica, foram retirados da amostra para não produzirem vieses.

O período analisado contempla as duas últimas avaliações trienais da CAPES: 2006-2008 e 20092011. Considerou-se a produção disseminada nestes periódicos nesse intervalo de tempo por compreenderem uma série mínima de cinco anos. 


\subsection{Hipóteses do estudo}

A relação entre periódicos contábeis brasileiros e as citações recebidas distribuídas pelos veículos de citação é o aspecto central desta investigação. Garfield (1972) apontou que há uma predominância das citações oriundas de estudos publicados em alguns periódicos preferenciais por parte dos usuários. Neste caso, a primeira hipótese desta pesquisa considera que há associação entre o periódico e as citações recebidas pelos artigos nele publicados. A expectativa é a negação da hipótese nula, por se considerar que há dependência entre valores esperados e observados. Se confirmada a significância estatística nessa relação, a associação será estudada à luz da análise de correspondência (ANACOR).

Para verificar a relação entre características dos artigos (periódico onde foi publicado, ano da publicação e idioma do artigo) e citações recebidas por veículo de citação (tipo de fonte onde foi citado), foram formuladas as hipóteses constantes na Tabela 2:

Tabela 2

\section{Hipóteses do estudo}

\begin{tabular}{cl}
\hline Hipóteses & \multicolumn{1}{c}{ Descrição } \\
\hline Hipótese 1 & Existe associação entre Periódico e Citaçães recebidas por veículo de citação \\
\hline Hipótese 2 & Existe associação entre Ano de publicação e Citações recebidas por veículo de citação \\
\hline Hipótese 3 & Existe associação entre Idioma do artigo e Citações recebidas por veículo de citação \\
\hline Hipótese 4 & Existe associação entre Idioma do artigo e País da citação \\
\hline
\end{tabular}

Quanto aos periódicos e respectivos artigos, a literatura aponta que há relação entre a concentração de citações nos primeiros anos após a publicação (Bianco, 2004; Amin \& Mabe, 2007), maior volume de citações de textos no idioma do país em pesquisas de países em desenvolvimento como o Brasil, com evidências de insularidade, ou seja, maior volume de citações apenas pelos pares do mesmo país (Ladle, Todd \& Malhado, 2012).

Para testar as hipóteses, foram elaboradas tabelas de contingência (referência cruzada) com a aplicação do teste de Qui-quadrado. A expectativa das hipóteses levantadas é que há diferença entre os valores esperados e observados nas relações entre cada característica e as citações recebidas, logo, que $\mathrm{H}_{0}$ seja negada.

\subsection{Procedimentos de coleta dos dados}

Para o registro inicial das observações, utilizou-se uma planilha eletrônica. As variáveis relativas aos artigos publicados foram: periódico, ano de publicação, número do fascículo, volume, número do artigo (ordem no sumário da edição), idioma do artigo e título do artigo.

As variáveis relativas às citações foram: veículo da citação, ano da citação, idioma, país, endereço eletrônico do texto que contém a citação e data de acesso.

Foram selecionados os artigos de todas as edições dos 4 periódicos mencionados, tendo como recorte temporal as duas últimas avaliações trienais do Sistema Qualis/CAPES (2006-2008 e 2009-2011). Os registros dos dados dos artigos ocorreram a partir dos sites dos periódicos, com análise de cada edição publicada dentro do período selecionado.

As observações contemplam as citações recebidas por 577 artigos, distribuídos em 78 fascículos, os quais representam a totalidade das edições publicadas dos periódicos que compõem a amostra no período analisado, disponíveis eletronicamente na fase de coleta, que ocorreu entre os meses de maio e junho de 2012.

Os registros das citações ocorreram a partir do acesso ao mecanismo de busca denominado Google Acadêmico (http://scholar.google.com.br), tendo como critério de filtro o título do artigo, com a expressão exata (entre aspas). A escolha do Google Acadêmico se deu por ser um mecanismo de busca de livre 
acesso a textos acadêmicos e indexador de citações acadêmicas, relativamente novo em estudos de produtividade da pesquisa (Lee et al., 2012). Além dos textos vinculados às citações apontadas pelo mecanismo, observaram-se as demais fontes apresentadas na busca e que, embora não vinculadas como citações, seus conteúdos enquadram-se nas categorias de registro. Foi verificado se o artigo constava nas referências do texto apresentado na busca, por meio da técnica de análise de conteúdo.

As variáveis são qualitativas nominais e foram categorizadas conforme Tabela 3:

Tabela 3

Categorias de classificação dos achados da pesquisa

\begin{tabular}{llll}
\hline \multicolumn{1}{c}{ Periódico } & Idioma da citação & País da citação & Veículo da citação \\
\hline - Contabilidade Vista \& Revista (CVista) & - Português & ・ Brasil & - Anais \\
- Revista Contabilidade \& Finanças (RC\&F) & - Inglês & ・ Outro país & - Artigo na web \\
- Revista de Contabilidade e Organizações (RCO) & • Espanhol & & - Dissertação \\
- Revista Universo Contábil (Universo) & - Outro & & - Monografia \\
& & & - Periódico \\
& & & - Projeto de Pesquisa \\
& & Tese \\
\hline
\end{tabular}

Para analisar a relação entre as características dos periódicos e as respectivas citações recebidas, utilizou-se o teste Qui-quadrado a partir de tabelas de contingência. Para identificar a relação principal do estudo (periódicos versus citações), foi utilizada a Análise de Correspondência (ANACOR). Os dados foram analisados com o uso do SPSS 20.

Ressalta-se que o estudo tem a restrição de a coleta ocorrer a partir de um mecanismo de busca na Web, cujas limitações de acesso podem diminuir a quantidade de citações efetivamente recebidas pelos artigos. Um exemplo é não reconhecer nas buscas o conteúdo da Biblioteca Digital de Teses e Dissertações da Universidade de São Paulo (USP) (http://www.teses.usp.br/), instituição que possui o PPG mais antigo em atividade na área contábil e a maior quantidade de Programas stricto sensu ativos no Brasil.

\section{Resultados e Discussão}

Foram registradas 1.655 observações, das quais 283 relativas a artigos que não receberam citação e 1.372 registros de citações classificadas nas categorias já mencionadas. As tabelas e gráficos apresentados neste tópico têm como fonte os dados da pesquisa.

\subsection{Análise descritiva dos dados}

A Tabela 4 a seguir apresenta a distribuição das observações de acordo com a situação dos artigos e das citações por ano e por veículo de citação. 
Tabela 4

Frequências dos artigos (citado ou não citado) por ano e das citações por ano e veículo de citação (2013)

\begin{tabular}{|c|c|c|c|c|c|c|c|c|c|c|c|c|c|}
\hline \multirow[b]{2}{*}{ Ano } & \multicolumn{5}{|c|}{ Artigos } & \multicolumn{8}{|c|}{ Citações } \\
\hline & Citados & $\%$ & $\begin{array}{c}\text { Não } \\
\text { citados }\end{array}$ & $\%$ & Total & Anais & $\begin{array}{l}\text { Artigo } \\
\text { na web }\end{array}$ & Dissert. & Monogr. & Periódico & $\begin{array}{l}\text { Proj. de } \\
\text { pesquisa }\end{array}$ & Tese & Total \\
\hline 2006 & 49 & 62,82 & 29 & 37,18 & 78 & 106 & 8 & 59 & 24 & 152 & 1 & 5 & 355 \\
\hline 2007 & 64 & 71,91 & 25 & 28,09 & 89 & 124 & 8 & 70 & 42 & 222 & 1 & 5 & 472 \\
\hline 2008 & 78 & 69,64 & 34 & 30,36 & 112 & 94 & 8 & 48 & 29 & 133 & 0 & 0 & 312 \\
\hline 2009 & 59 & 55,66 & 47 & 44,34 & 106 & 44 & 9 & 34 & 12 & 67 & 0 & 3 & 169 \\
\hline 2010 & 36 & 36,73 & 62 & 63,27 & 98 & 18 & 3 & 9 & 5 & 19 & 0 & 1 & 55 \\
\hline 2011 & 8 & 8,51 & 86 & 91,49 & 94 & 3 & 0 & 0 & 2 & 4 & 0 & 0 & 9 \\
\hline Total & 294 & 50,95 & 283 & 49,05 & 577 & 389 & 36 & 220 & 114 & 597 & 2 & 14 & 1.372 \\
\hline
\end{tabular}

Os resumos das frequências absolutas e relativas, bem como a média de citações por artigo por ano, são apresentados na Tabela 5 a seguir.

Tabela 5

Frequências absolutas (Fi) e relativas da quantidade de artigos, quantidade de citações e média de citações por artigo de cada ano (2013)

\begin{tabular}{|c|c|c|c|c|c|c|c|c|c|c|c|c|c|}
\hline \multirow{2}{*}{ Descrição } & \multicolumn{2}{|c|}{2006} & \multicolumn{2}{|c|}{2007} & \multicolumn{2}{|c|}{2008} & \multicolumn{2}{|c|}{2009} & \multicolumn{2}{|c|}{2010} & \multicolumn{2}{|c|}{2011} & \multirow{2}{*}{ Total } \\
\hline & $\mathbf{F i}$ & $\%$ & $\mathbf{F i}$ & $\%$ & $\mathbf{F i}$ & $\%$ & $\mathbf{F i}$ & $\%$ & $\mathbf{F i}$ & $\%$ & $\mathbf{F i}$ & $\%$ & \\
\hline Quantidade de artigos & 78 & 13,52 & 89 & 15,42 & 112 & 19,41 & 106 & 18,37 & 98 & 16,98 & 94 & 16,29 & 577 \\
\hline Quantidade de citações & 355 & 25,87 & 472 & 34,40 & 312 & 22,74 & 169 & 12,32 & 55 & 4,01 & 9 & 0,66 & 1.372 \\
\hline Média de citações por artigo & 4,55 & & 5,30 & & 2,79 & & 1,59 & & 0,56 & & 0,10 & & 2,38 \\
\hline
\end{tabular}

Os dados apresentados nas Tabelas 4 e 5 permitem observar que os artigos publicados em 2007 apresentaram o maior volume absoluto de citações, com 472, e a média de citações por artigo no ano foi a maior do período analisado, com 5,30 citações. Embora fosse esperado um maior volume de citações dos artigos mais antigos, aqueles publicados no primeiro ano não foram os que receberam mais citações. $\mathrm{O}$ número de citações menor nos artigos mais recentes pode ser atribuído à maturação, ou seja, ainda não foram tão lidos quanto os mais antigos, por isso são menos citados, como sugerem Amin e Mabe (2007).

Quanto às citações por veículo, a maioria ocorreu em periódicos, seguidas de anais de eventos. Vão ao encontro do que a literatura descreve como canais oficiais de disseminação do conhecimento: os eventos, como espaços de discussão, e os periódicos, como meio das publicações definitivas. Os 283 artigos que não receberam citação equivalem a 49,05\% do total de artigos publicados, com destaque para 2011, com média de apenas 0,1 citação por artigo. A RCO surgiu em 2007, por este motivo o total de artigos em 2006 é menor.

Outro dado importante é que cerca de $30 \%$ dos artigos com mais de 03 anos de publicados não apresentaram citações, sendo que em 2006 esse percentual atingiu 37,08\%. Esses trabalhos não produziram ressonância, ou seja, embora publicados em periódicos que pertencem atualmente aos estratos superiores do Qualis/CAPES, não despertaram interesse da comunidade acadêmica no seu uso como base para outros estudos. Os de 2006, em particular, já atingiram a meia vida citada mencionada Amim e Mabe (2007) sem terem recebido citação.

A Tabela 6 apresenta o detalhamento das observações de acordo com o periódico, o ano e o veículo em que ocorreu a citação: 
Tabela 6

Frequências dos artigos (citado ou não citado) por periódico e das citações por periódico e veículo de citação (2013)

\begin{tabular}{|c|c|c|c|c|c|c|c|c|c|c|c|c|c|}
\hline \multirow[b]{2}{*}{ Period. } & \multicolumn{5}{|c|}{ Artigos } & \multicolumn{8}{|c|}{ Citações } \\
\hline & Citados & $\%$ & $\begin{array}{c}\text { Não } \\
\text { citados }\end{array}$ & $\%$ & Total & Anais & $\begin{array}{l}\text { Artigo } \\
\text { na web }\end{array}$ & Dissert. & Monogr. & Periódico & $\begin{array}{l}\text { Proj. de } \\
\text { pesquisa }\end{array}$ & Tese & Total \\
\hline CVista & 65 & 46,76 & 74 & 53,24 & 139 & 55 & 5 & 19 & 21 & 78 & 1 & 2 & 181 \\
\hline $\mathrm{RC} \& \mathrm{~F}$ & 109 & 75,69 & 35 & 24,31 & 144 & 267 & 24 & 153 & 59 & 397 & 1 & 9 & 910 \\
\hline RCO & 42 & 39,62 & 64 & 60,38 & 106 & 19 & 0 & 13 & 8 & 38 & 0 & 0 & 78 \\
\hline Universo & 78 & 41,49 & 110 & 58,51 & 188 & 48 & 7 & 35 & 26 & 84 & 0 & 3 & 203 \\
\hline Total & 294 & 50,95 & 283 & 49,05 & 577 & 389 & 36 & 220 & 114 & 597 & 2 & 14 & 1.372 \\
\hline
\end{tabular}

Os resumos das frequências absolutas e relativas, bem como a média de citações por artigo por periódico, são apresentados na Tabela 7 a seguir.

Tabela 7

Frequências absolutas e relativas da quantidade de artigos, quantidade de citações e média de citações por artigo de cada periódico (2013)

\begin{tabular}{|c|c|c|c|c|c|c|c|c|c|}
\hline \multirow{2}{*}{ Descrição } & \multicolumn{2}{|c|}{ CVista } & \multicolumn{2}{|c|}{ RC\&F } & \multicolumn{2}{|c|}{ RCO } & \multicolumn{2}{|c|}{ Universo } & \multirow{2}{*}{ Total } \\
\hline & $\mathbf{F i}$ & $\%$ & $\mathbf{F i}$ & $\%$ & $\mathbf{F i}$ & $\%$ & $\mathbf{F i}$ & $\%$ & \\
\hline Quantidade de artigos & 139 & 24,09 & 144 & 24,96 & 106 & 18,37 & 188 & 32,58 & 577 \\
\hline Quantidade de citações & 181 & 13,19 & 910 & 66,33 & 78 & 5,69 & 203 & 14,80 & 1.372 \\
\hline Média de citações por artigo & 1,30 & & 6,32 & & 0,74 & & 1,08 & & 2,38 \\
\hline
\end{tabular}

Os achados apresentados nas Tabelas 6 e 7 evidenciam que os artigos publicados pela RC\&F e citados em periódicos foram os que apareceram com maior frequência. Os artigos da RC\&F receberam 66,33\% do total de citações coletadas. Em seguida, a UNIVERSO com 14,80\%, a CVISTA com 13,19\% e, por fim, a RCO com 5,69\%. Embora a RCO tenha apresentado a menor participação percentual no total de citações recebidas, os periódicos com maiores percentuais de artigos não citados são a CVISTA e a UNIVERSO, com percentuais superiores a 50\%. Ressalta-se, entretanto, que estes volumes sofrem influência da quantidade de artigos publicados mais recentemente, os quais se encontram na janela Índice de Imediaticidade e, por consequência, tendem a ser menos citados por estarem em maturação.

Os dados desagregados das citações por artigo mostraram que dois artigos da RC\&F, publicados nas edições 40 e Especial 2006, apresentaram a maior quantidade individual de citações, com 51 ocorrências. $\mathrm{Na}$ CVISTA o artigo mais citado foi publicado em 2007 no vol. 1, n. ${ }^{\circ} 18$, e recebeu 14 citações. Um artigo do vol. 2, n. ${ }^{\circ} 2$ da RCO, publicado em 2008, apresentou 8 citações, tendo sido o mais citado deste periódico. Já na UNIVERSO, este número foi de 16 citações recebidas por um artigo publicado no vol. 1, n. ${ }^{\circ} 2$, em 2006.

Os dados confirmam a RC\&F como o periódico que recebeu o maior volume de citações, com média por artigo de 6,32, o que representa mais que o dobro da média geral.

\subsection{Testes das hipóteses e a análise de correspondência}

A Tabela 8 apresenta os testes de Qui-quadrado da relação entre as variáveis que delineiam as hipóteses deste estudo: 
Tabela 8

Resumo dos testes Qui-quadrado das hipóteses do estudo

\begin{tabular}{llccccc}
\hline \multirow{2}{*}{ Hipótese } & \multirow{2}{*}{ Relação } & \multicolumn{2}{c}{ Sumário dos Casos } & \multicolumn{3}{c}{ Teste $\chi^{2}$} \\
\cline { 3 - 7 } & & Casos Válidos & $\%$ & Valor & GL & Sig. \\
\hline Hipótese 1 & Periódico versus Veículo de citação & 1.655 & 100,00 & $316,767^{a}$ & 21 &, 000 \\
\hline Hipótese 2 & Ano de publicação versus Veículo de citação & 1.655 & 100,00 & $583,229^{a}$ & 35 &, 000 \\
\hline Hipótese 3 & Idioma do artigo versus Veículo de citação & 1.655 & 100,00 & $107,664^{a}$ & 14 &, 000 \\
\hline Hipótese 4 & Idioma do artigo versus País da citação & 1.655 & 100,00 & $172,131^{a}$ & 4 &, 000 \\
\hline
\end{tabular}

Como se percebe na Tabela 8, os resultados dos testes mostraram-se significativos nas relações analisadas, com valores de probabilidade menores que o alfa estipulado 5\% - rejeição da hipótese nula. A relação entre periódico e veículo de citação, objeto central deste estudo, apresentou significância estatística e justifica a aplicação da ANACOR.

As características Ano de publicação do artigo e Idioma do artigo guardam relação com a quantidade de citações recebidas, como demonstram os resultados do teste das Hipóteses 2 e 3 . Significa que sugerem a existência de uma relação de dependência entre as categorias e as citações por veículo de citação, pois os valores observados são estatisticamente significativos quando comparados com os valores esperados.

O Idioma do artigo possui associação com o país da citação e está de acordo com o apresentado por Ladle, Todd \& Malhado, (2012) quanto à prevalência de citações de textos no idioma nativo, ou seja, textos em português mais citados por brasileiros.

A partir da análise de correspondência, buscou-se identificar a associação entre o periódico e o veículo de citação. Os resultados são apresentados na Tabela 9 a seguir:

Tabela 9

Sumário da análise de correspondência da relação Periódico versus citações (2013)

\begin{tabular}{|c|c|c|c|c|c|c|c|c|}
\hline \multirow{3}{*}{ Dimensão } & \multirow{3}{*}{$\begin{array}{l}\text { Valores } \\
\text { Singulares }\end{array}$} & \multirow{3}{*}{ Inércia } & \multirow{3}{*}{$\begin{array}{c}\text { Chi } \\
\text { Quadrado }\end{array}$} & \multirow{3}{*}{ Sig. } & \multicolumn{2}{|c|}{ Proporção de Inércia } & \multicolumn{2}{|c|}{$\begin{array}{c}\text { Confiança de Valores } \\
\text { Singulares }\end{array}$} \\
\hline & & & & & \multirow{2}{*}{ Contado por } & \multirow{2}{*}{ Cumulativo } & \multirow{2}{*}{$\begin{array}{l}\text { Desvio- } \\
\text { padrão }\end{array}$} & \multirow{2}{*}{$\begin{array}{c}\text { Correlação } \\
2\end{array}$} \\
\hline & & & & & & & & \\
\hline 1 &, 430 & , 185 & & & 966 & 966 & ,021 &,- 007 \\
\hline 2 & ,063 & ,004 & & & 021 & 986 & ,024 & \\
\hline 3 &, 051 & ,003 & & &, 014 & 1,000 & & \\
\hline Total & & 191 & 316,767 &, $000^{\mathrm{a}}$ & 1,000 & 1,000 & & \\
\hline
\end{tabular}

a. 21 graus de liberdade

O teste Qui-Quadrado confirmou que há associação entre as variáveis Periódico e Veículo de citação, que contém o volume de citações recebidas. A Dimensão 1 explica $96,6 \%$ da inércia total e a Dimensão 2 explica apenas $2,1 \%$. Este resultado sugere que a Dimensão 1 é mais importante para explicar o comportamento dos dados. Ressalta-se que a Dimensão 3, por apresentar contribuição marginal de apenas $1,4 \%$ da inércia total, não foi considerada.

Os escores das linhas na ANACOR permitiram observar que a RC\&F foi quem mais contribuiu para a formação da inércia da Dimensão 1, com 40,7\%, seguida da RCO, com 25,5\%. A Dimensão 2, por sua vez, teve 71,9\% de contribuição da CVISTA para a formação da inércia. Os dados demonstraram que a RC\&F foi quem mais contribuiu para explicar o comportamento dos dados, é quem mais influencia na formação da principal dimensão. A análise das dimensões nos pontos de linha e de coluna são demonstradas a seguir: 


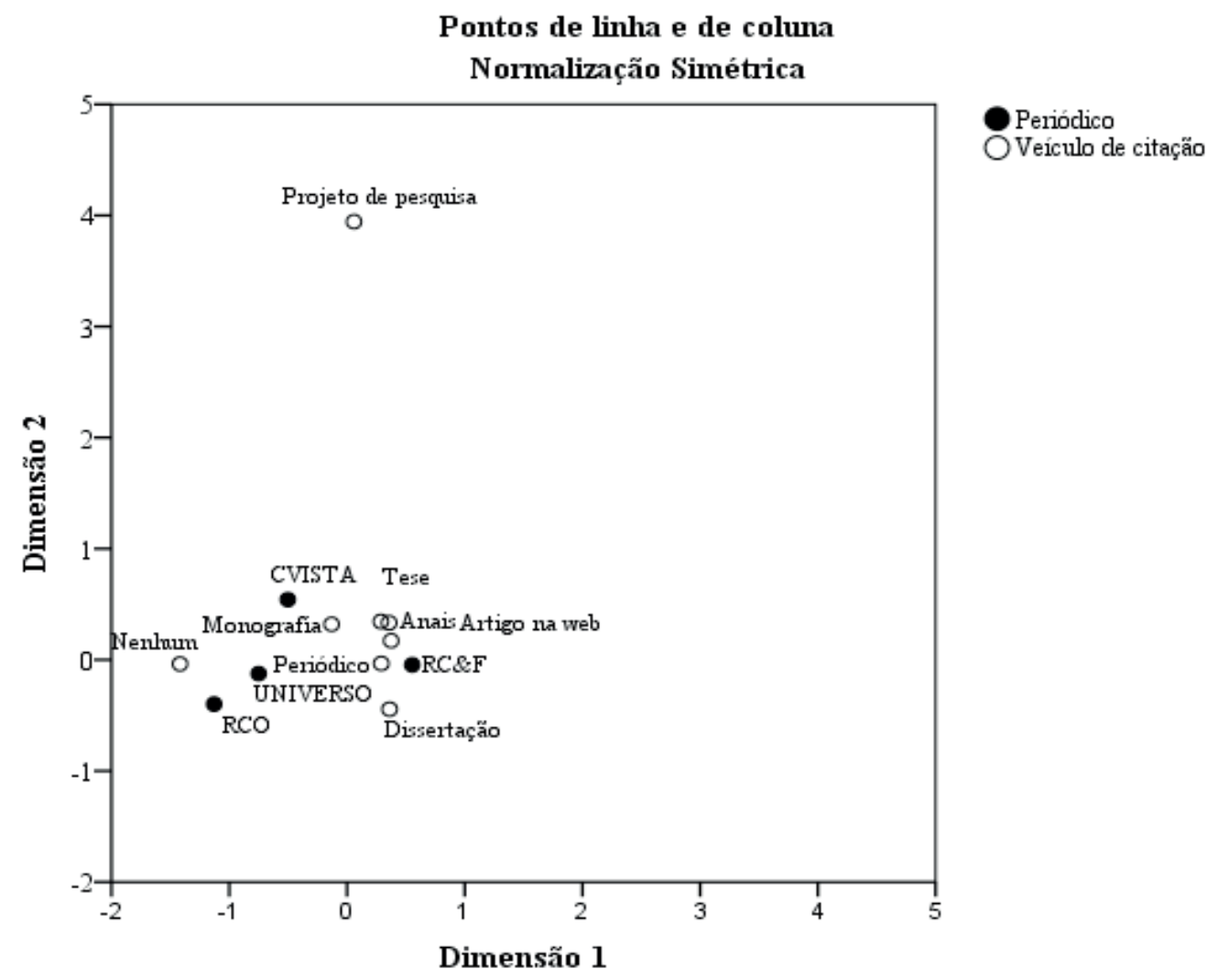

Figura 2. Pontos de linha por Periódico e pontos de coluna por Veículo de citação (2012)

Na Figura 2, que representa os pontos de coluna e de linha, pode-se perceber que a RC\&F se destaca na Dimensão 1, que é a que melhor explica o comportamento dos dados. Os outros periódicos se concentram na Dimensão 2, com menor poder de explicação. Em torno da RC\&F estão a maior parte dos veículos de citação. Apenas Projeto de pesquisa figurou na Dimensão 2, sem associação direta com os periódicos. Já a categoria Nenhuma, que representa os artigos não citados, está mais associada aos periódicos RCO e UNIVERSO.

Na ANACOR, a análise dos resíduos permite perceber a associação entre cada par de variáveis. Para Fávero, Belfione, Silva \& Chan (2009, p. 279), os valores negativos dos resíduos padronizados “[...] indicam menor associação (similaridade).” entre os pares. No caso em estudo, a normalização utilizada foi a simétrica, aplicada a situações em que "[...] há o interesse em examinar as diferenças ou similaridades entre as duas variáveis simultaneamente." (Fávero, Belfione, Silva \& Chan, 2009, p. 289). Os resíduos constam na Tabela 10, a seguir:

Tabela 10

Resíduos padronizados da relação Periódico versus Veículo de citação (2013)

\begin{tabular}{lccccccccc}
\hline & \multicolumn{1}{c}{ Veículo de citação } \\
\cline { 2 - 11 } Periódico & Nenhum & Anais & $\begin{array}{c}\text { Artigo } \\
\text { na web }\end{array}$ & Dissertação & Monografia & Periódico & $\begin{array}{c}\text { Projeto de } \\
\text { pesquisa }\end{array}$ & Tese \\
\hline CVista & 4,6 &,- 6 &,- 2 & $-2,6$ & -8 & $-1,5$ & 1,2 &,- 1 \\
\hline RC\&F & $-10,0$ & 3,0 & -8 & 2,4 &,- 8 & 3,0 &,- 1 &, 4 \\
\hline RCO & 8,1 & $-2,5$ & $-1,8$ & $-1,4$ &,- 6 & $-1,8$ &,- 4 & $-1,1$ \\
\hline Universo & 7,7 & $-3,0$ &, 1 & $-1,0$ & 1,0 & $-2,7$ &,- 6 &, 2 \\
\hline
\end{tabular}


Da análise dos resíduos padronizados da relação Periódico versus Veículo de citação, presentes na Tabela 10, pode-se observar que os valores positivos indicam:

- A RCO, seguida da UNIVERSO e da CVISTA, foram os periódicos que apresentaram maior associação com artigos que não receberam citações. $\mathrm{O}$ valor negativo da RC\&F demonstra menor associação desta com os artigos não citados.

- A CVISTA apresentou maior associação com o veículo Projeto de pesquisa, embora tenha se destacado também no veículo Monografia (o qual inclui o Trabalho de Conclusão de Curso - TCC)

- A RC\&F apresenta maior associação nos veículos: Anais, Artigo na web, Dissertação e Periódico.

- A UNIVERSO foi quem apresentou maior associação com o veículo Monografia, tendo relação positiva também com os veículos Artigo na web e Tese.

Esta análise dos resíduos padronizados ratifica que há associação entre a variável Periódico e as citações, representadas por meio da variável Veículo de citação.

\subsection{Fatores de impacto dos periódicos analisados}

A partir do modelo do cálculo proposto por Amin e Mabe (2007), foram calculados os fatores de impacto dos periódicos analisados relativos a 2008 a 2011. Os detalhes do cálculo, considerando o modelo do JCR, são apresentados nas Tabelas 11 e 12:

Tabela 11

Fatores de impacto dos periódicos analisados para os anos de 2008 e 2009 (2013)

\begin{tabular}{|c|c|c|c|c|c|c|c|c|c|c|}
\hline \multirow{3}{*}{ Periódico } & \multicolumn{5}{|c|}{2008} & \multicolumn{5}{|c|}{2009} \\
\hline & \multicolumn{3}{|c|}{ Artigos publicados } & \multirow{2}{*}{$\begin{array}{l}\text { Citações } \\
\text { recebidas } \\
\text { em } 2008\end{array}$} & \multirow{2}{*}{$\begin{array}{l}\text { Fator de } \\
\text { Impacto }\end{array}$} & \multicolumn{3}{|c|}{ Artigos publicados } & \multirow{2}{*}{$\begin{array}{l}\text { Citações } \\
\text { recebidas } \\
\text { em } 2009\end{array}$} & \multirow{2}{*}{$\begin{array}{l}\text { Fator de } \\
\text { Impacto }\end{array}$} \\
\hline & 2006 & 2007 & Total & & & 2007 & 2008 & Total & & \\
\hline CVista & 25 & 24 & 49 & 7 & 0,143 & 24 & 24 & 48 & 9 & 0,188 \\
\hline $\mathrm{RC} \& \mathrm{~F}$ & 32 & 34 & 66 & 84 & 1,273 & 34 & 26 & 60 & 84 & 1,400 \\
\hline $\mathrm{RCO}$ & 0 & 8 & 8 & 1 & 0,125 & 8 & 26 & 34 & 2 & 0,059 \\
\hline Universo & 21 & 23 & 44 & 9 & 0,205 & 23 & 36 & 59 & 14 & 0,237 \\
\hline Total & 78 & 89 & 167 & 101 & 0,605 & 89 & 112 & 201 & 109 & 0,542 \\
\hline
\end{tabular}

Tabela 12

Fatores de impacto dos periódicos analisados para os anos de 2010 e 2011 (2013)

\begin{tabular}{|c|c|c|c|c|c|c|c|c|c|c|}
\hline \multirow{3}{*}{ Periódico } & \multicolumn{5}{|c|}{2010} & \multicolumn{5}{|c|}{2011} \\
\hline & \multicolumn{3}{|c|}{ Artigos publicados } & \multirow{2}{*}{$\begin{array}{l}\text { Citações } \\
\text { recebidas } \\
\text { em } 2010\end{array}$} & \multirow{2}{*}{$\begin{array}{l}\text { Fator de } \\
\text { Impacto }\end{array}$} & \multicolumn{3}{|c|}{ Artigos publicados } & \multirow{2}{*}{$\begin{array}{l}\text { Citações } \\
\text { recebidas } \\
\text { em } 2011\end{array}$} & \multirow{2}{*}{$\begin{array}{l}\text { Fator de } \\
\text { Impacto }\end{array}$} \\
\hline & 2008 & 2009 & Total & & & 2009 & 2010 & Total & & \\
\hline CVista & 24 & 24 & 48 & 20 & 0,417 & 24 & 24 & 48 & 32 & 0,667 \\
\hline $\mathrm{RC} \& \mathrm{~F}$ & 26 & 22 & 48 & 106 & 2,208 & 22 & 14 & 36 & 31 & 0,861 \\
\hline $\mathrm{RCO}$ & 26 & 24 & 50 & 19 & 0,380 & 24 & 24 & 48 & 22 & 0,458 \\
\hline Universo & 36 & 36 & 72 & 29 & 0,403 & 36 & 36 & 72 & 33 & 0,458 \\
\hline Total & 112 & 106 & 218 & 174 & 0,798 & 106 & 98 & 204 & 118 & 0,578 \\
\hline
\end{tabular}


Os fatores de impacto foram calculados a partir da quantidade de artigos publicados e de citações identificadas com o uso do Google Acadêmico. A fim de melhor comparar, a Figura 3 a seguir representa os fatores ano a ano, bem como o fator médio dos periódicos.

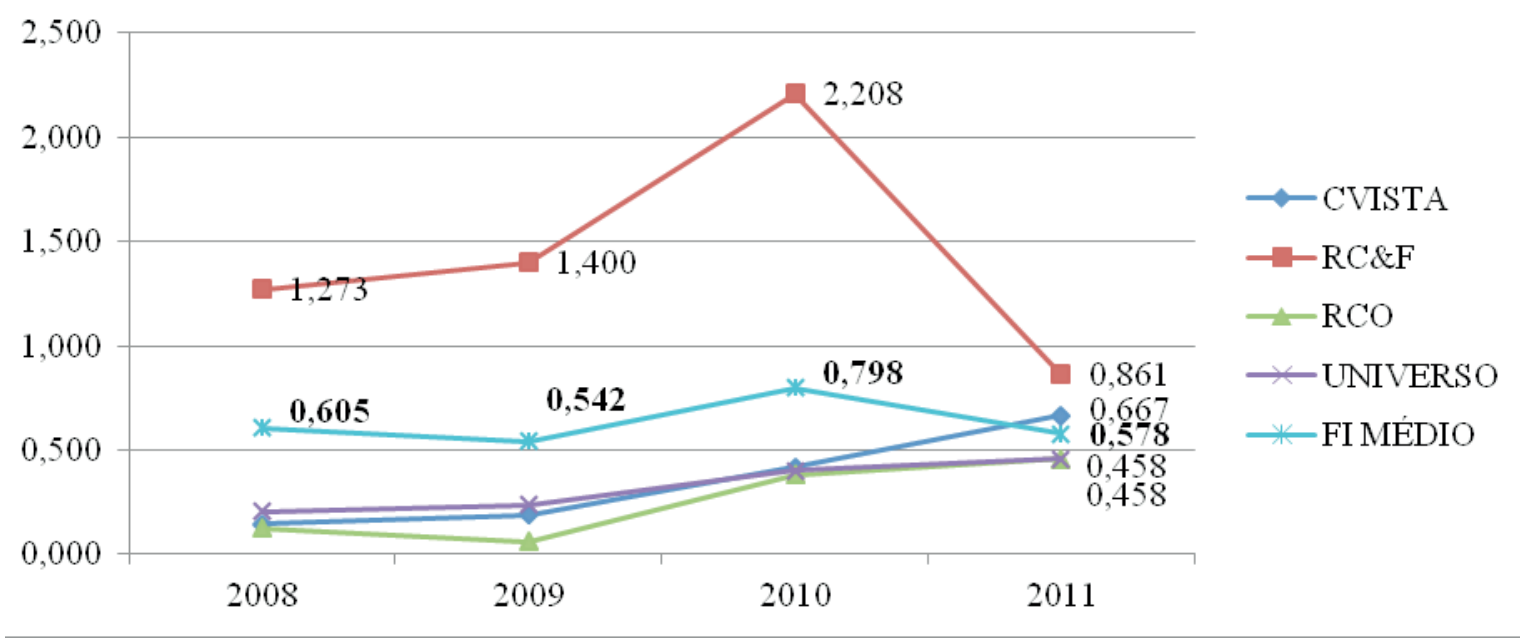

Figura 3. Fatores de impacto dos periódicos analisados (2008 a 2011).

A RC\&F apresentou o maior fator de impacto, entretanto, com uma diminuição no último ano. Os FI dos periódicos RCO e UNIVERSO no ano de 2011 foram idênticos; embora este último tenha mais citações em números absolutos, também apresentou número maior de artigos publicados por edição.

O comportamento médio dos FI nos três primeiros anos foi superior ao dobro daqueles obtidos pelos periódicos CVISTA, RCO e UNIVERSO. O fator da RC\&F no período foi significativamente mais alto em relação aos demais, entretanto, houve uma queda expressiva entre 2010 e 2011, tendo passado de 2,208 para 0,861, embora continue sendo o periódico com o maior FI no ano de 2011.

Os resultados confirmam a intensidade da ressonância da RC\&F na produção científica da área contábil do Brasil. Embora ainda inferiores, os fatores de impacto dos três demais periódicos têm crescido ao longo do período analisado e se aproximaram daquele do periódico com maior fator em 2011.

\section{Conclusões}

Estudos sobre a ressonância da pesquisa científica podem servir de alerta à comunidade acadêmica contábil do Brasil, em virtude da possibilidade potencial de fornecer subsídios para informar, por meio de indicadores, possíveis acertos e erros cometidos por pesquisadores. Estes podem se materializar na seleção de temáticas, na sustentação teórica escolhida, no caminho metodológico e nas reflexões produzidas.

Nesse sentido, este estudo identificou os 4 periódicos contábeis brasileiros publicados pelos Programas de Pós-Graduação stricto sensu ou por Instituições que os oferecem, classificados nos estratos superiores do Qualis/ CAPES. Foram levantadas as citações recebidas pelos 577 artigos publicados nos 78 fascículos desses periódicos ao longo de 6 anos. Das 1.655 observações efetuadas, 283 são relativas a artigos que não receberam citação.

Os achados permitiram observar que periódico onde foi publicado, ano da publicação do artigo, idioma do artigo e país da citação foram características estatisticamente significativas quando cruzadas com as citações recebidas. A significância estatística confirma que as frequências observadas diferem-se das esperadas, ou seja, estatisticamente há dependência entre as características do periódico/artigo e o número de citações recebidas. Situação semelhante se verificou entre o idioma do artigo e o país onde foi publicado o texto com a citação. Além disso, cerca de metade dos artigos publicados não receberam citação, o que demonstra que a relevância ainda não se apresenta em parte dos artigos publicados. 
A análise de correspondência buscou observar a ressonância da produção publicada nos periódicos, dada pela relação entre os periódicos e as citações recebidas pelos estudos publicados nesses. Esta relação também foi significativa e os resultados da ANACOR demonstram que a RC\&F foi o periódico mais citado, sendo aquele que mais pesa na Dimensão 1, a qual melhor explica a variabilidade dos dados. Os resíduos padronizados demonstram que alguns veículos de citação têm prevalência, dependendo do periódico citado.

Os achados permitem concluir que a ressonância da produção científica analisada pode ser considerada baixa, com média de 2,38 citações por artigo no período, elevado volume de artigos não citados e concentração de citações em um periódico, sendo que a variabilidade dos dados tem relação com as características dos artigos/periódicos. O maior fator de impacto, calculado com base nos achados, foi obtido pela RC\&F, com 2,208 em 2010 - periódico que mais contribuiu para a Dimensão 1 e que foi preponderante em relação aos periódicos estudados. Os FI da RCO $(0,458)$ e UNIVERSO $(0,458)$ foram os menores, portanto, estes influenciam em menor intensidade a produção científica. A CVISTA, com 0,667, apresentou fator ligeiramente maior a estas, entretanto, tem diminuído a distância em relação à RC\&F.

Tais dados respondem à questão do estudo e atingem aos objetivos da pesquisa. Também estão de acordo com os achados apresentados por Garfield (1972) de que há uma concentração das citações em determinados periódicos e, consequentemente, nos artigos publicados nestes.

O interesse da comunidade acadêmica pela produção científica contábil brasileira pode ser fortalecido a partir da análise da essência das temáticas discutidas. É necessário refletir sobre as possíveis consequências econômicas e sociais da produção científica em Contabilidade. Nesse sentido, a ressonância se manifesta como marca de utilidade e as respostas e/ou novos questionamentos dela decorrentes são evidências da sua capacidade de transformar e comunicar. A produção científica tenderá a ser desinteressante e incoerente com o contexto contemporâneo se a discussão apresentada não contemplar a relevância social, ou seja, se torna um monólogo inócuo.

Caberia ao pesquisador rever as questões que direcionam sua produção de maneira a considerar a relevância do que deve ser produzido. Os estudos são inovadores e atuais? São discutidas situações práticas capazes de contribuir para a reflexão e crítica das práticas contábeis? Há perspectiva de se encontrar elementos teóricos que fomentem novas questões de pesquisa ou trazer possíveis respostas às demandas sociais contemporâneas? Ou ainda, os periódicos contábeis brasileiros estão publicando estudos considerados úteis e relevantes pelos seus usuários?

A investigação desenvolvida utilizou o fator de impacto, uma medida de avaliação reconhecida internacionalmente, para analisar os periódicos nacionais vinculados aos programas de pós-graduação da área contábil. Embora relevantes no contexto brasileiro, não se identificou análise de tais periódicos a partir do modelo proposto por Garfield (1972).

De fato, apesar das limitações e críticas ao fator de impacto, deve-se ressaltar que este é apenas uma das exterioridades significativas a serem discutidas em matéria de produção científica. De maneira distinta dos estudos anteriores aqui mencionados, a ressonância não tem sido foco dos estudos que tratam sobre a publicação científica no âmbito contábil brasileiro. Acredita-se que ela traz consigo uma percepção acerca dos efeitos que tal produção tem causado quando partilhada e conhecida pela comunidade científica.

Sugere-se que outros estudos possam ser realizados a fim de ampliar a percepção da ressonância das publicações classificadas em outros estratos do Qualis/CAPES, como forma de conhecer outra visão do perfil da produção científica da área contábil brasileira disseminada em seus periódicos acadêmicos. 


\section{Referências}

Amara, N \& Landry, R. (2012). Counting citations in the field of business and management: why use google scholar rather than the web of science. Scientometrics. 93(3), p. 553-581.

Amin, M, \& Mabe, M. (2007). Impact factors: use and abuse. Perspectives in Publishing, n. 1, oct. 2000. Reissued with minor revisions October.

Bianco, A. C. (2004). Fator de impacto: boletim do editor? (editorial). Arquivos Brasileiros de Endocrinologia \& Metabologia, 48(3). Recuperado em 09 julho, 2012, de http://www.scielo.br/pdf/abem/ v48n3/a02v48n3.pdf.

Carmona, S. (2011). In pursuance of successful research. European Accounting Review, 20(1), p. 1-5.

Campello, B. S. (2000). Organizações como fonte de informação. In: Campello, B. S., Cendón, B. V. \& Kremer, J. M. (orgs.). Fontes de informação para pesquisadores e profissionais. Belo Horizonte: Ed. UFMG. p. 35-48.

Capes. Coordenação de Aperfeiçoamento de Pessoal de Nível Superior. (2012). Comunicado no 002/2012: área de administração, ciências contábeis e turismo: atualização do webqualis da área. Recuperado em 08 maio, 2012 de http://qualis.capes.gov.br/arquivos/avaliacao/webqualis/criterios2010_2012/ Criterios_Qualis_2011_27.pdf.

Chan, L., \& Costa, S. (2005). Participation in the global knowledge commons: challenges and opportunities for research dissemination in developing countries. New Library World. 106(1210/1211), p. 141-163.

Christensen, J. (2011). Good analytical research. European Accounting Review, 20(1), p. 41-51.

Czarniawska, B. (2011). Successful research: in whose eyes? European Accounting Review. 20(1), p. 53-55.

Fávero, L. P., Belfione, P., Silva, F. L., \& Chan, B. L. (2009) Análise de dados: modelagem multivariada para a tomada de decisões. Rio de Janeiro: Elsevier.

Flick, U. (2009). Qualidade na pesquisa qualitativa. Trad. Roberto Cataldo Costa. Consultoria, supervisão e revisão técnica Dirceu da Silva. Porto Alegre: Artmed (Coleção Pesquisa Qualitativa, coordenada por Uwe Flick).

Garfield, E. (1972). Citation analysis as a tool in journal evaluation. Essays of an Information Scientist, Vol 1, p. 527-544, 1962-73. Recuperado em 04 julho, 2012 de http://www.elshami.com/Terms/I/impact\%20factor-Garfield.pdf.

King, D. A. (2004). The scientific impact of nations: what different countries get for their research spending. Nature. v. 430. Recuperado em 03 julho, 2012 de http://webarchive.nationalarchives.gov.uk/+/ http://www.berr.gov.uk/files/file11959.pdf.

Kuramoto, H. (2006). Informação científica: proposta de um novo modelo para o Brasil. Ciência da Informação. Brasília, 35(2), p. 91-201.

Ladle, R. J., Todd, P. A., \& Malhado, A. C. M. (2012). Assessing insularity in global science. Scientometrics. 93(3), p. 745-750. Recuperado em 09 julho, 2012 de http://dx.doi.org/10.1007/s11192-012-0703-z.

Lee, T. H., Yap, C. S., Lim, Y. M., \& Tam, C. L. (2012). Accounting researchers in Asia Pacific: a study on publication productivity and citation analysis. Asian Journal of Finance \& Accounting, 4(1), p.132-150.

Martins, G. de A. \& Theóphilo, C. R. (2007). Metodologia da investigação científica para ciências sociais aplicadas. São Paulo: Atlas.

Meadows, A. J. A. (1999). A comunicação científica. Brasília: Briquet de Lemos.

Miranda, D. B. de, \& Pereira, M. de N. F. (1996). O periódico científico como veículo de comunicação: uma revisão de literatura. Ciência da Informação, Brasília, 25(3), p.375-382. Recuperado em 22 março, 2013 de http://revista.ibict.br/ciinf/index.php/ciinf/article/viewFile/462/421. 
Mueller, S. P. M. (1999). O círculo vicioso que prende os periódicos nacionais. DataGramaZero - Revista de Ciência da Informação, 0. Recuperado em 09 julho, 2012 de http://eprints.rclis.org/bitstream/10760/6189/1/Art_04.htm.

Mueller, S. P. M. (2000). O periódico científico. In: Campello, B. S., Cendón, B. V., \& Kremer, J. M. (orgs.). Fontes de informação para pesquisadores e profissionais. Belo Horizonte: Ed. UFMG. p. 73-96.

Nelson, R. R. (1959). The simple economics of basic scientific research. Journal of Political Economy. 67(3), p. 297-306. Published by: The University of Chicago PressStable. Recuperado em03 julho 2012 de http://www.jstor.org/stable/1827448.

Ohlson, J. A. (2011). On successful research. European Accounting Review, 20(1), p. 7-26.

Packer, A. L. (2001). The SciELO model for electronic publishing and measuring of usage and impact of latin american and caribbean scientific journals. Proceedings of the Second ICSU-UNESCO International Conference on Electronic Publishing in Science, Paris 20-23 February. Recuperado em 04 julho, 2012 de http://eos.wdcb.ru/eps2/unesco.tex/pdf/packerfn.pdf.

Pendlebury, D. A. (2009). The use and misuse of journal metrics and other citation indicators. Arch. Immunol. Ther. Exp, Philadelphia, 57(1), p.1-11. doi: 10.1007/s00005-009-0008-y.

Pinto, A. C., \& Andrade, J. B. de. (1999). Fator de impacto de revistas científicas: qual o significado deste parâmetro? Química Nova, São Paulo, 22(3), p.448-453. Recuperado em 22 março, 2013 de http:// www.scielo.br/pdf/qn/v22n3/1101.pdf.

Pinto, L. A. (2008). Cientometria: é possível avaliar a qualidade da pesquisa científica? (Editorial) - Scientia Medica, 18(2), p.64-65. Recuperado em 04 maio, 2012 de http://revistaseletronicas.pucrs.br/ojs/ index.php/scientiamedica/article/view/3637/3016.

Rosenstreich, D., \& Wooliscroft, B. (2009). Measuring the impact of accounting journals using google scholar and the g-index. The British Accounting Review, 41(4), p. 227-239.

Strehl, L. (2005). O fator de impacto do ISI e a avaliação da produção científica: aspectos conceituais e metodológicos. Ciência da Informação, 34(1), p.19-27. Recuperado em 02 julho, 2012 de http://www. scielo.br/pdf/\%0D/ci/v34n1/a03v34n1.pdf.

Tahai, A. \& Rigbsy, J. (1998). Information processing using citation to investigate journal influence in accounting. Information Processing \& Management. 34(2), p. 341-359.

Vanclay, J. K. (2012). Impact factor: outdated artefact or stepping-stone to journal certification? Scientometrics, 92(2), p. 211-238

Vilhena, V., \& Crestana, M. F. (2002). Produção científica: critérios de avaliação de impacto. Revista da Associação Médica Brasileira, São Paulo, 48(1), p.1-25. Recuperado em 22 março, 2013 de http:// www.scielo.br/pdf/ramb/v48n1/a22v48n1.pdf.

Wang, C. Y. (2012). The intellectual structure of modern accounting research: concepts, theories and relationships. African Journal of Business Management, 6(23), p. 6860 - 6866.

Yamamoto, O. H., Menandro, P. R. M., Koller, S. H., Lo Bianco, A. C., Hutz, C. S., Bueno, J. L. O., \& Guedes, M. C. (2002). Avaliação de periódicos científicos brasileiros da área de psicologia. Ciência da Informação, 31(2), p. 163-177. 